\title{
RARS-related autosomal recessive hypomyelinating leukodystrophy
}

INSERM

\section{Source}

INSERM. (1999). Orphanet: an online rare disease and orphan drug data base. RARSrelated autosomal recessive hypomyelinating leukodystrophy. ORPHA:438114

A rare, genetic leukodystrophy characterized by developmental delay, increased muscle tone leading later to spasticity, mild ataxia, nystagmus, dysarthria, intentional tremor, and mild intellectual disability. Brain imaging reveals supratentorial and infratentorial hypomyelination. 